\title{
Research on Robustness of Emergency Transportation System of Materials Based on the Three-layer Interdependent Network
}

\author{
Boyu Feng ${ }^{1, \mathrm{a}}$,Lin Zhou ${ }^{2, \mathrm{~b}}$,Zhihao Zhang ${ }^{* 3, \mathrm{c}}$,Lei Zhang ${ }^{4, \mathrm{~d}}$ \\ ${ }^{1}$ Equipment Management and UAV Engineering College , Airforce Engineering University Xian,Shanxi ,China \\ ${ }^{2}$ Air and Missile Defense College, Airforce Engineering University Xian,Shanxi ,China \\ ${ }^{3}$ Air Traffic Control and Navigation College, Airforce Engineering University Xian,Shanxi, China \\ ${ }^{4}$ Equipment Management and UAV Engineering College, Airforce Engineering University Xian,Shanxi ,China
}

\begin{abstract}
The ability to transport materials under emergency conditions is one of the important capabilities of the country to respond to crises. In order to study the ability of the emergency material transportation system to resist external damage, this paper builds a three-layer interdependent network model based on capacity-load under the analysis of the basic characteristics of the system, and conducts random attacks on the network, studies the relationship between overall robustness and transportation flow of this three-layer network . In addition, the robustness of the three sub-networks is studied, which provides a theoretical basis for constructing a reasonable and robust emergency material transportation system.
\end{abstract}

\section{Introduction}

The outbreak of COVID-19 in early 2020 is a test of the modern governance system and governance capabilities of countries around the world. Due to the sudden increase in demand for some materials and the shutdown of production, the transportation of various countries has been greatly affected, and the materials cannot be transported and equipped in time, which affects the speed of the country's handling of emergencies to a certain extent. It can be seen that it is very important to establish a complete national emergency transportation network that can resist external shocks.

In the research of material emergency transportation network, the theory and method of dependent network, which have been widely concerned by scholars in recent years, are powerful scientific tools. The aggregation of several similar nodes in the emergency transportation network of materials can be considered as a complex subnetwork, and several sub-networks coupled with each other are collectively called interdependent network [1]. The study found that the dependent network can describe the real world more accurately than the general singlelayer complex network [2] [3]. Since Buldyrev and others put forward the concept of dependent networks, scholars all over the world have achieved a lot of fruitful results in this field in the past ten years [4]. However, due to the dual attributes of information transmission network and military emergency, the material emergency transportation network should focus on its sensitivity to disturbance response and the robustness of the network. The existing network model mainly has the following two limitations:

(1) Most of the existing dependent network models are two-layer networks, so the number of network levels cannot meet the requirements of the material emergency transportation network network.

(2) Most models do not consider the problem of interlayer flow in the network, and cannot truly reflect the impact of changes in the flow of materials and equipment of the emergency transportation network on the network performance.

\section{Modeling of Material emergency transportation network}

According to the needs of emergency transportation of materials, in view of the problems of diversified situations in emergency and wartime, diversified resource requirements, and the status of the wide application of emerging technologies in emergency situations, a threelayer interdependent network model of "resourcestransport-user" (RTU model) is constructed. The network model does not exist independently, and the interdependent network is formed by the dependence of the nodes between the layers of the network.

\subsection{Interdependent network model}

There is a single connection between each layer of the RTU three-layer dependent network model. The main characteristics of the network are extracted, and the RTU three-layer dependent network model shown in the figure below is constructed. 


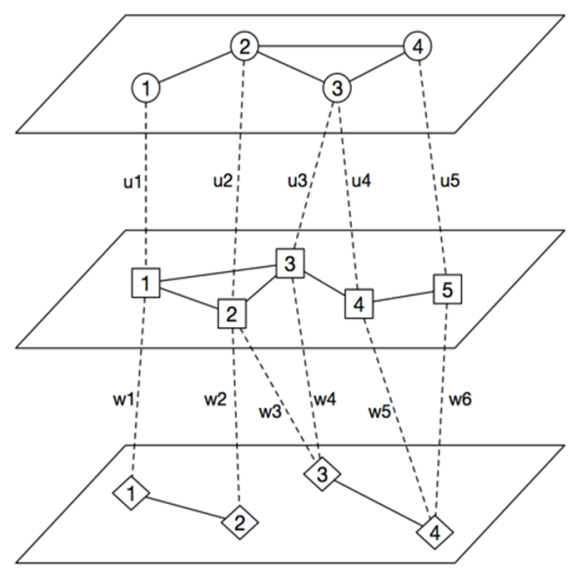

Fig1. RTU three-layer dependent network

Connect nodes in different networks based on resources and users and the transportation relationship between them, where $\underline{t}_{1}(i=1,2,3 \ldots n)$ represents the inter-layer dependency between the user network and the resource transmission network; $w_{i}(i=1,2,3 \ldots m)$ represents the dependency between the resource transmission network and materials. $\underline{u}_{i}, w_{i} \in[0,11$, when $\overline{E_{1} \text { OR } w_{i}}=0$, it indicates that there is no inter-layer dependency between the two nodes linked by this edge, and the edge can be deleted. When $t_{1}$ OR $w:=1$, the interlayer dependence relationship between the two points connected by the edge is the strongest, and the failure of a certain point can cause the complete failure of the connected point.

The network model can be described by the set $\mathrm{G}(\mathrm{N}, \mathrm{L}, \mathrm{W})$, where $\mathrm{N}$ is the total number of nodes in the RTU network; L is the set of internal and external coupling edges in the dependent network; $\mathrm{W}$ is the coupling matrix.

\subsection{Subnet description}

The three-layer subnets in the RTU network are all physical networks of social organizations. They have common characteristics similar to the Internet and the Internet of Things, that is, some nodes have a large number of links, and most nodes have only a few connections, and their degree distribution shows power-law form, generally expressed as $\mathrm{P}(k) \sim k^{-\gamma}$ [5].

When generating the network, we follow the following principles:

(1) The node scales of the three-tier subnet are the same, that is $N^{A}=N^{B}=N^{C}$.

(2) The network growth mechanism is used to establish a subnet, that is, nodes are set in the initial state, and then a new node is introduced at each step, and the nodes introduced each time are randomly connected to $\mathrm{n}$ original nodes, of which $n \in\left[\sigma_{s} x_{3}\right]$.

(3) Follow the priority connection mechanism, that is, the connection probability between a new node and an existing node $\mathrm{i}$ satisfies the following formula:

$$
\Pi_{i}=\frac{k_{i}+1}{\sum_{j} k_{j}+1}
$$

\section{RTU network cascading failure model}

\subsection{M-L model}

Considering the actual relationship between the node entities that make up each sub-layer of the RTU network, this paper selects the classic M-L model proposed by Motter et al., that is, the "load-capacity" model, and defines the capacity $\mathrm{C}$ of node I as follows:

$$
\mathrm{C}_{i}=(1+\beta) L_{i}(0)
$$

In the above formula, $\beta \geqslant 0$, which is the tolerance coefficient. The larger the value, the stronger the network's ability to resist cascade. But in actual problems, the robustness of the network will increase the cost of constructing the network, and the $\beta$ value needs to be controlled within a certain range. It is meaningful to compare the survivability of the network when other parameters are different under a certain $\beta$ value [6].

\subsection{Robustness measure of three-layer dependent network}

After the cascading failure has spread in the three-layer dependent network, the damage degree of the network is defined as follows:

$$
F=\frac{N_{f}^{A}+N_{f}^{B}+N_{f}^{C}}{N^{A}+N^{B}+N^{C}}
$$

In formula (3), $N_{f}^{A} 、 N_{f}^{B} 、 N_{f}^{C}$ is the number of failed nodes in networks A, B, and C, respectively. After being attacked, the smaller the value of $\mathrm{F}$, the stronger the anti-destructiveness of the three-layer dependent network [7].

In addition, the maximum connected subgraph ratio after being attacked is also an important robustness measure of dependent complex networks, which is defined as follows:

$$
G=\frac{N_{g}^{A}+N_{g}^{B}+N_{g}^{C}}{N^{A}+N^{B}+N^{C}}
$$

Among them, $N_{g}^{A} 、 N_{g}^{B} 、 N_{g}^{C}$ is the number of nodes in the largest connected subgraph in the single-layer network after the failure of networks A, B, and C. After being attacked, the larger the value of $G$, the stronger the anti-destructiveness of the three-layer dependent network.

\section{Simulation experiment and analysis}

According to the M-L model mentioned above, the threelayer dependent network is modeled, and then the dependent network is randomly attacked to cause the network to cascade failures. Observe the influence of network flow and capacity coefficient on the robustness of the three-layer dependent network. 


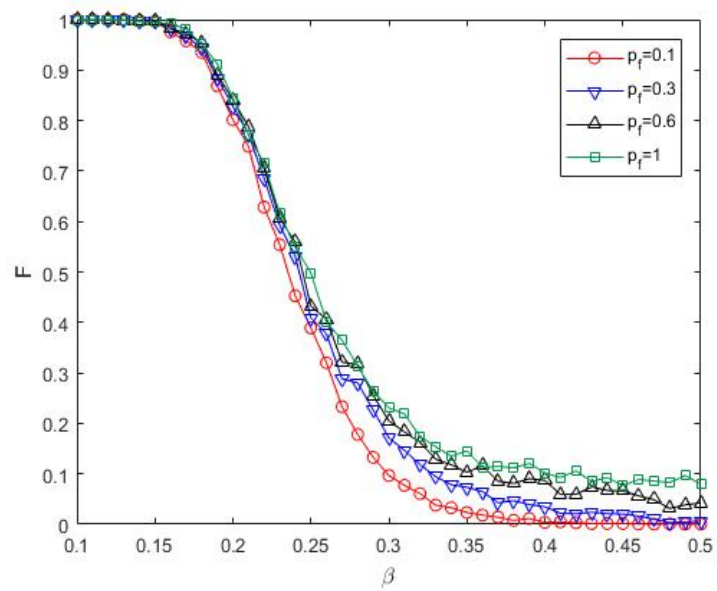

Fig2. The number of remaining nodes after the network is attacked under different flow

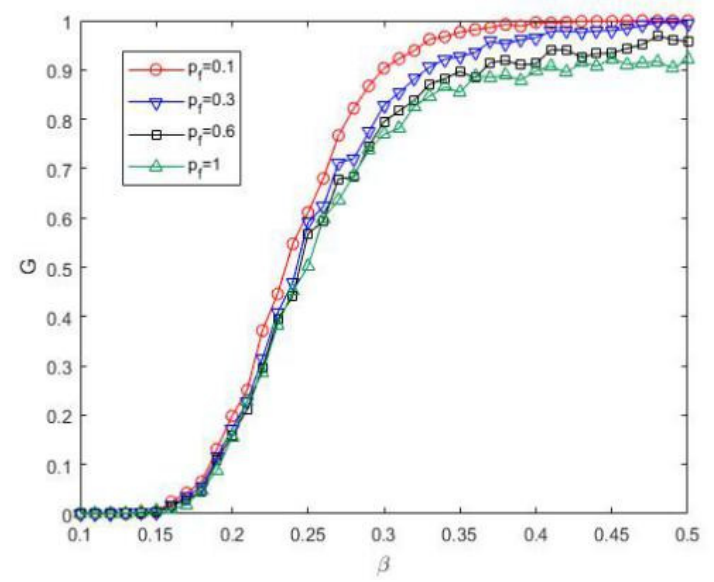

Fig3. The largest connected subgraph scale after the network is attacked under different flow

It can be seen from the curve in Figure 2 that as the tolerance coefficient $\beta$ increases, the four curves in the figure all show a downward trend, which means that the degree of network damage $\mathrm{F}$ is gradually reduced, that is, the robustness of the network is gradually enhanced. When the network flow $P_{f}$ is set to $0.1,0.3,0.6$, and 1 , as the $P_{f}$ increases, the robustness of the network gradually becomes weaker, which means that the larger the network flow, the more cascading failures of the threelayer dependent network are likely to occur. Figure 3 uses the largest connected subgraph to characterize the robustness of the network, and the conclusions obtained are consistent with Figure 2.

On the basis of inspecting all network nodes, observe the law of the robustness of the three subnets of the dependent network with the tolerance coefficient. The network flow is taken as 0.5 during the simulation, and the results are shown in Figure 4 and Figure 5.

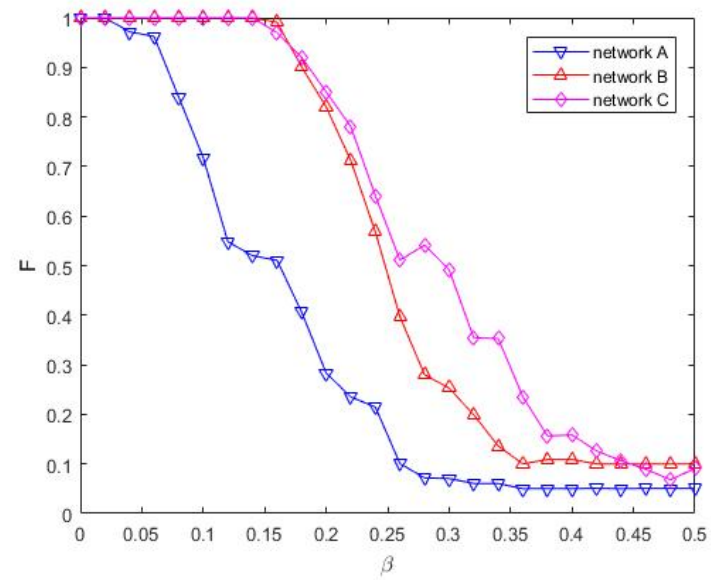

Fig4. Ratio of failed nodes in three sub-networks

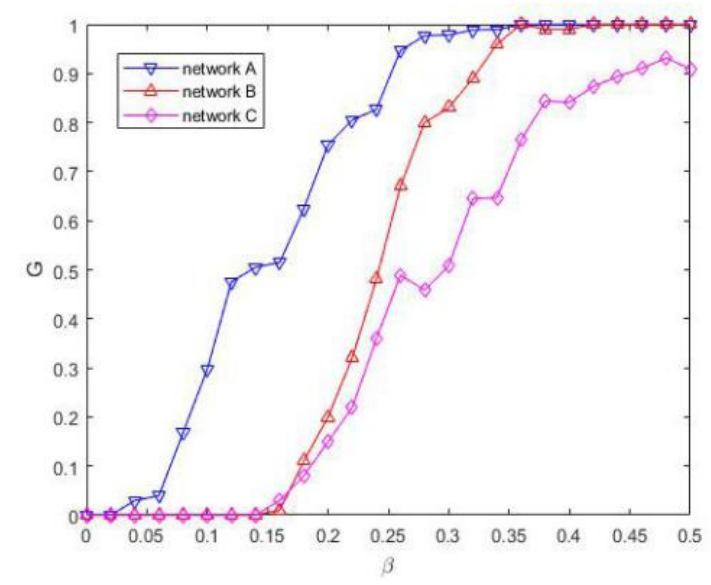

Fig5. Largest connected sub-graph scale after network failure

It can be seen from Figure 4 and Figure 5 that as the tolerance coefficient $\beta$ increases, the robustness of the three sub-networks gradually increases, but in contrast, the robustness of the first layer network (network A) is more obvious It is better than the second layer network (network $\mathrm{B}$ ) and the third layer network (network C). When $\beta$ is less than 0.25 , the robustness of network $B$ and network $C$ is roughly the same. When the value of $\beta$ is greater than 0.25 , the robustness of the second layer network is better than that of the third layer network. The robust performance of the three-layer sub-network shows the result of $\mathrm{A}>\mathrm{B}>\mathrm{C}$ as a whole.

Analyze the reason, when the second layer network receives a fault from the first layer network, the node is considered to be removed when its node loses the dependent connection between the first and second layer networks. After a certain node is removed, its load will follow The rules of the ML model are passed to neighboring nodes. In this way, in the second layer network, there are faults caused by the failure of the first layer network nodes at the same time, and there are also cascading failures of the failure transmission of the nodes of this layer, so the failure rate is significantly higher than that of the first layer network. In the same way, the failure rate of the third-tier network is greater than that of the second-tier network, but because the failure principles of nodes in the second and third-tier networks are basically the same, the rate difference is not obvious. 


\section{Conclusion}

This article analyzes the importance of a smooth and robust national logistics transportation network under emergency conditions, puts forward the main characteristics of the material emergency transportation network, and establishes a three-tier material emergency transportation network. Through simulation experiments, it is found that when the network traffic gradually increases, the overall survivability of the three-layer dependent network gradually weakens, which conflicts with the demand for a large amount of material transportation in emergency situations, which requires a reasonable deployment of the flow value on the emergency transportation network. When the three-layer sub-networks are separately observed, it is found that from the first-layer network to the third-layer network, the degree of cascading failures of the sub-networks is gradually serious, which shows that the impact of the failure propagation to the lower network is greater than the upper network. The above research conclusions can provide a theoretical basis for the construction of emergency logistics transportation network and load distribution, thereby enhancing the survivability of the emergency logistics system and ensuring the country's logistics and transportation safety in war and emergency.

\section{References}

1. Baker, Brent H., Graham, Tim.Network Failure.[J].National Review,1998,50,(11).

2. Kleinberg,Jon,Sandler,Mark,Slivkins,Aleksandrs..Ne twork Failure Detection and Graph Connectivity[J].SIAM Journal on Computing,2008,38,(4).

3. Melih Madanoglu, Gary J. Castrogiovanni.Franchising proportion and network failure[J].Small Business Economics,2018,50,(4).

4. Jilong Zhong, FengMing Zhang, Shunkun Yang, Daqing Li.Restoration of interdependent network against cascading overload failure[J].Physica A: Statistical Mechanics and its Applications,2019,514,(Complete).

5. Avash Das, Bhaskar Roy, Guido Schwarzer, Michael G. Silverman, ... Saumya Das.Comparison of treatment options for depression in heart failure: A network meta-analysis[J].Journal of Psychiatric Research,2019,108,(Complete).

6. Qian Zhu, Zhiliang Zhu, Yi Qi, Hai Yu, Yanjie Xu.Optimization of cascading failure on complex network based on NNIA[J].Physica A: Statistical Mechanics and its Applications,2018,501,(Complete).

7. Dong Zhou \& Ahmed Elmokashfi.Network recovery based on system crash early warning in a cascading failure model[J].Scientific Reports,2018,1,(1). 